\title{
Integration and installation of the CMS pixel barrel detector
}

\author{
Hans-Christian Kästli *i \\ Paul Scherrer Institut \\ E-mail: hans-christian.kaestliepsi.ch
}

A 66 million pixel detector has been installed in 2008 into the CMS experiment at CERN. The development and construction time took more than 10 years. In this paper the assembly of the barrel detector is described. A simple but effective method to accomplish a survey of the module positions during assembly is discussed. Furthermore the insertion and commissioning of the CMS pixel barrel detector which took place in July 2008 is illustrated.

17th International Workshop on Vertex detectors

July 28-August 1, 2008

Utö Island, Sweden

\footnotetext{
*Speaker.

† On behalf of the CMS pixel barrel group
} 


\section{Introduction}

The innermost part of the CMS detector at the Large Hadron Collider (LHC) near Geneva is a 66 million channel pixel tracking system surrounded by a 10(12) layer silicon strip tracker in the central (forward/backward) region. The pixel detector consists of 3 barrel layers at 4.4, 7.3 and $10.2 \mathrm{~cm}$ radius and two end disks on each side at $\mathrm{z}= \pm 35.5$ and $\pm 46.5 \mathrm{~cm}$ [1]. This article describes the integration, insertion and commissioning of the barrel part (BPIX). In section 2 the mechanical layout is described. For a more complete overview of the BPIX system the reader is referred to [2]. Section 3 and 4 explain the integration of the detector and its survey, which has been done simultaneously. Finally, in section 5 a first summary of the commissioning phase is given.

\section{Detector overview}

The BPIX system consists of two mechanically and electrically independent parts, the left and right half detector. The two parts have been assembled and tested separately before they were transported to CERN. Figure 1 shows half of the BPIX detector inside an installation cassette.

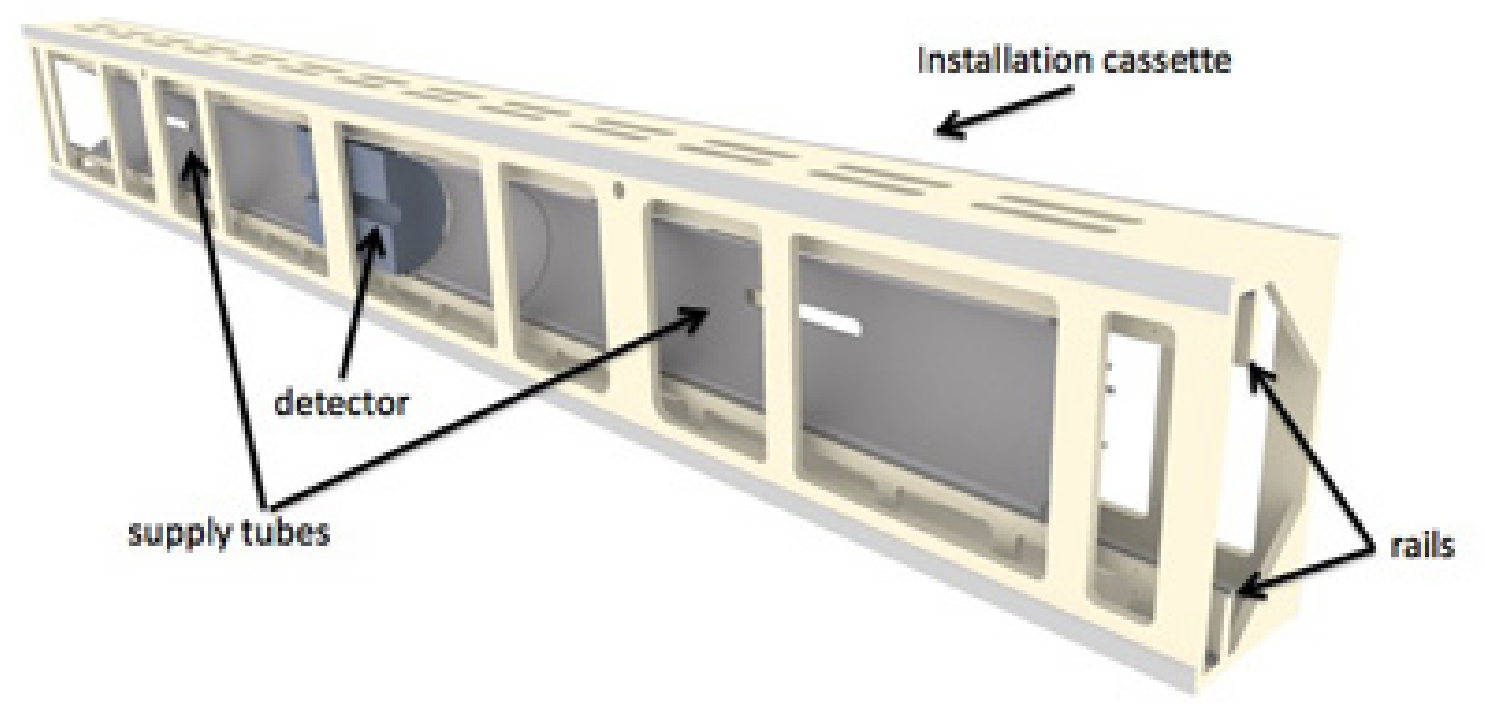

Figure 1: Drawing of one half of the barrel pixel system inside the installation cassette.

\subsection{Modules}

Figure 2 shows the components of a pixel barrel module. It consists of a $285 \mu \mathrm{m}$ thick n-on$\mathrm{n}$ type sensor bump-bonded to 16 or 8 readout chips. A high density interconnect (HDI) with a controller chip is glued on top of the sensor and wirebonded to the readout chips. Power is brought in through copper cladded aluminum wires of $0.25 \mathrm{~mm}$ diameter soldered to the HDI. A Kapton signal cable is glued and wirebonded to the HDI. This so called bare module is then glued onto a Si-nitride base plate with mounting screw holes in it. The assembly of the 672 full and 96 half modules is described in [3]. 


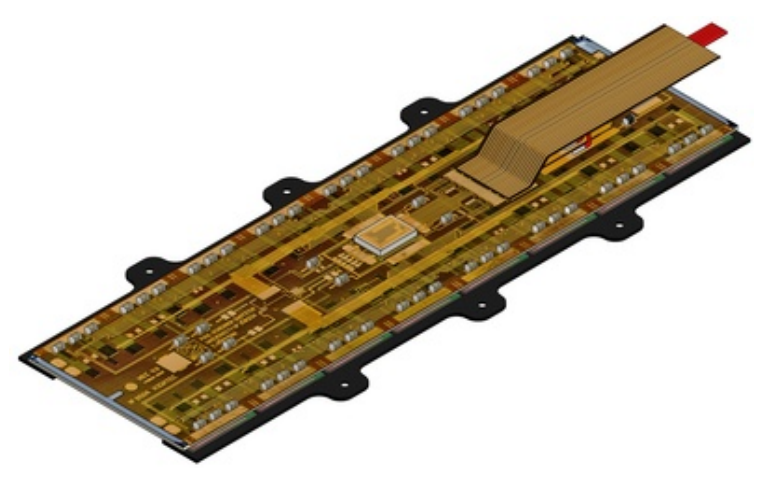

Figure 2: Drawing of a CMS pixel barrel full module.

\subsection{Barrel mechanics}

The barrel detector is a $530 \mathrm{~mm}$ long structure. The basic unit is a half layer. It is composed of $26 \mathrm{~mm}$ wide and $300 \mu \mathrm{m}$ thin carbon fibre sheets glued onto aluminum cooling pipes with $250 \mu \mathrm{m}$ wall thickness. The 3 layers have 10,16 and 22 facets respectively. They are glued on alternating sides of the cooling pipes. Each facet forms a ladder holding 8 modules. Screw nuts with $0.5 \mathrm{~mm}$ thread are glued into the carbon structure for module mounting.

The end flanges are made with AIREX foam sandwiched between carbon fibre plates and glass fibre reinforced edges. Layer one is screwed onto layer two and layer two onto layer 3. The end flange of layer three holds $0.5 \mathrm{~mm}$ thin 6 layer PCBs where the signal and power cables of all the modules from all layers are connected. The module cables are fixed at the end of the ladder with a tiny cable clamp. Figure 3 shows half of the barrel pixel detector fully assembled.

\subsection{Supply tube}

Each half barrel is connected on both sides with a $2.2 \mathrm{~m}$ long supply tube. It houses the electrooptical conversion boards for both the digital (DOH) control links and the analog (AOH) readout links. The AOH (DOH) contains 6 (4) laser diodes with $2 \mathrm{~m}$ long optical fibres glued to them. For logistics reason and for the sake of flexibility during the assembly, all the $\mathrm{AOH}$ and $\mathrm{DOH}$ boards are identical and hence the fibres have the same length.These fibres are laid in sector wise (one sector is 1/16th of one side) in the supply tube and covered by a thin aluminum coated GRP shielding. The slack management of the fibres is a tedious work and critical in terms of yield. At the far end of the supply tube optical connectors link the single fibres with fibre bundles of 12 fibres each. These bundles form $\approx 0.6 \mathrm{~m}$ long pig tails which then connect to the services at a patch panel near the end of the silicon tracker volume.

\section{Detector integration}

Each module has to be adapted to its particular position on the mechanical structure. This involves several steps:

- Setting the geographical address of each module by removing wire bonds according to a pre-defined address scheme. 


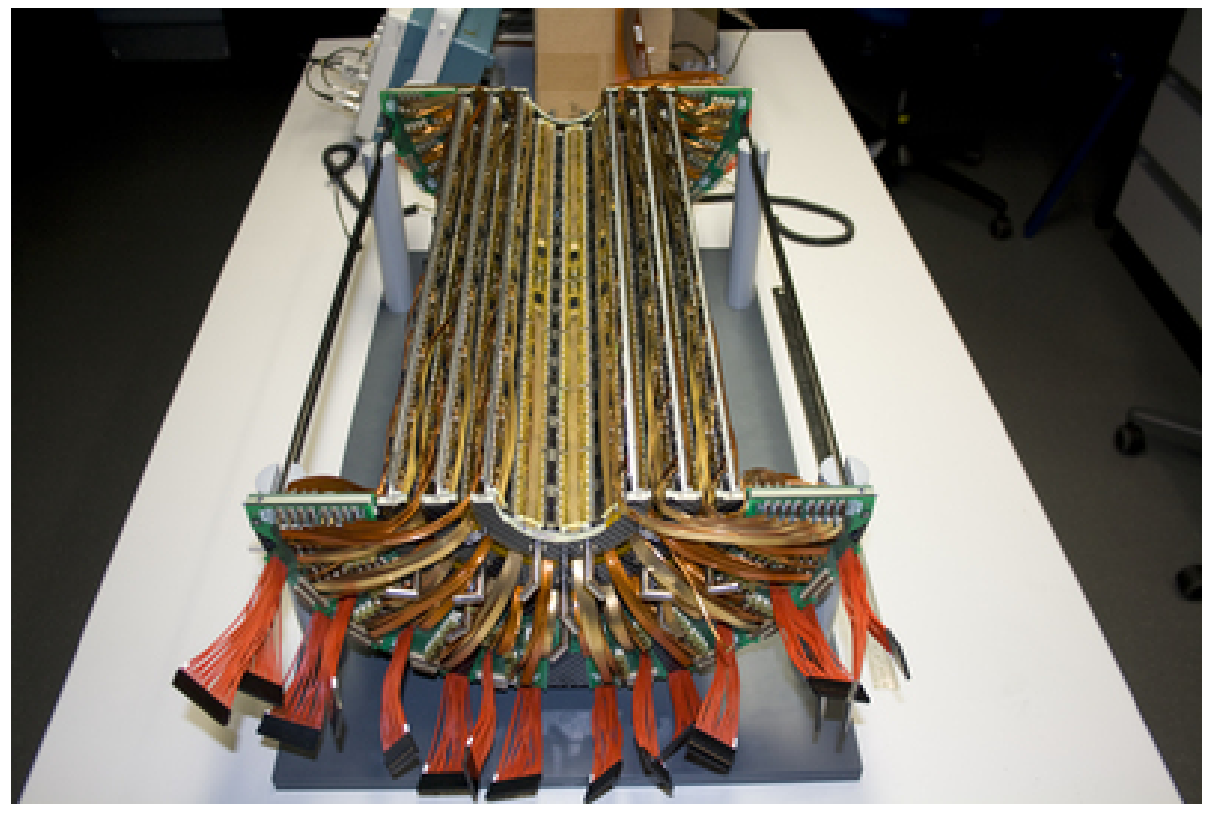

Figure 3: Half of the barrel pixel detector. All modules are mounted and connected to a thin 4 layer PCB on the end flange. The red cables bring the power from the supply tube to the end ring PCB.
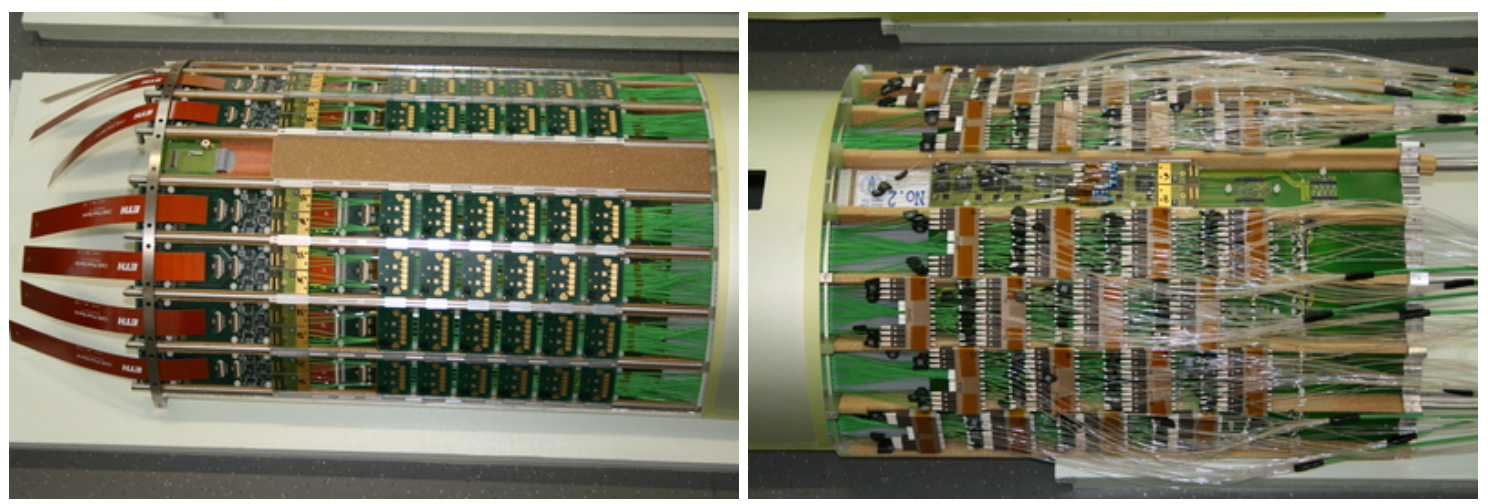

Figure 4: BPIX supply tube. Left: detector side. In each sector the board holding 6 analog and 1 digital opto hybrid daughterboards can be seen. The Kapton signal cables connecting to the detector end flange are visible on the far left side. Right: off detector side. The supply tube is completely covered with optical connectors.

- Shortening and re-connectorization of the power cables within $\approx 2 \mathrm{~mm}$ of the predetermined length. A 1:1 mock-up was used to measure the cable lengths to this precision.

- Shortening and bending of the signal cables. A special bending tool was designed to have a well defined bending radius and exact positions of the bending.

- Stitching together signal and power cable in well defined positions. This is important since towards the end flanges stacks of four cable pairs build up. 

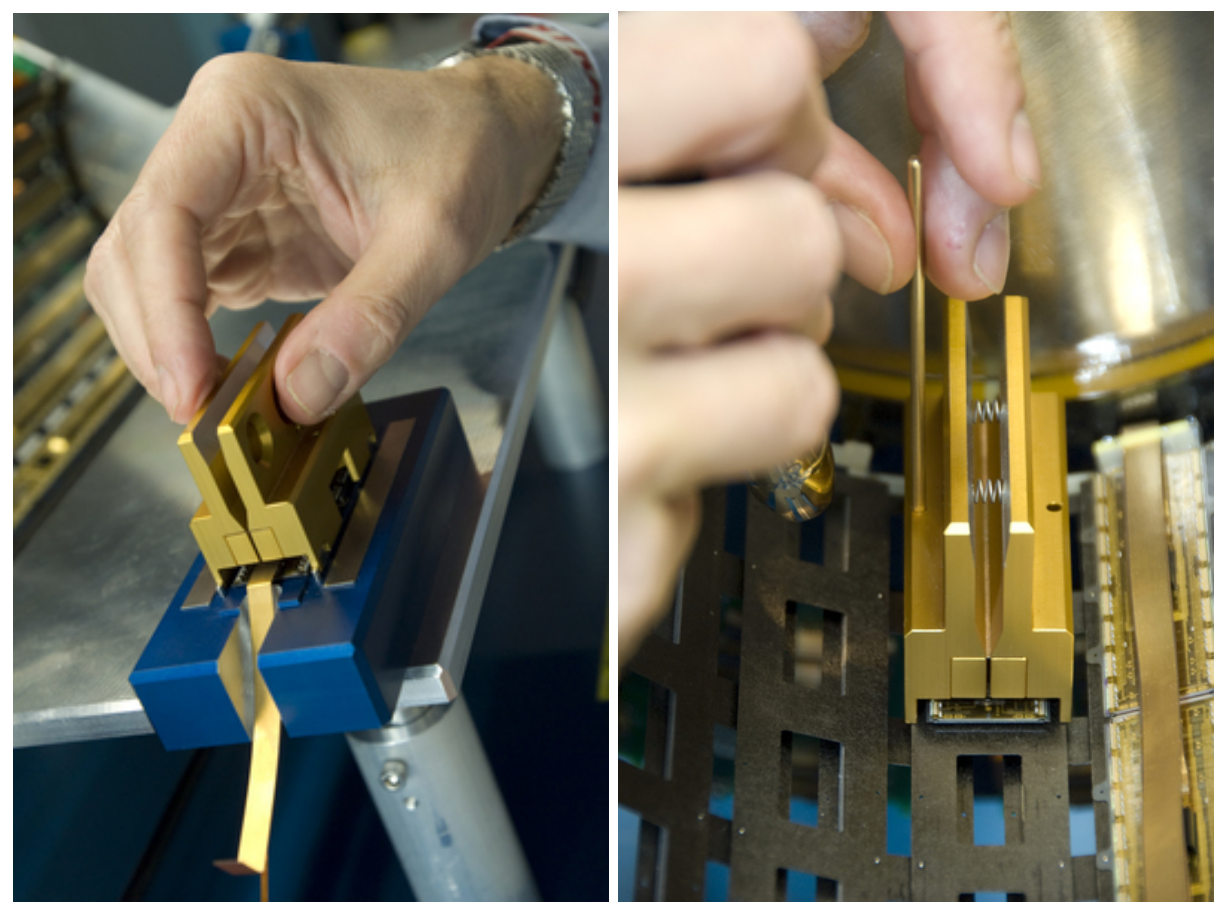

Figure 5: Module mounting tool. Left: the module is put on the jig (dark blue block) where it can be picked up with the mounting clamp. Right: the mounting clamp completely protects the module until the screws are tightened on the support structure.

During all these manipulations the module is protected in a special box. The module is fixed at the base stripes such that wire bonds and other delicate structures cannot be touched. Only the cables stick out.

To mount the module on the support structure, it has to be transferred into a mounting tool. This is shown in figure 5. In a first step, the module is put on a jig (blue). Two small pins fit through the mounting screw holes of the module and hold in place. The actual mounting tool is a clamp (golden) with a $100 \mu \mathrm{m}$ thin stainless steel sheet that grips under the module base plate and fixes its position in the tool. The clamp has a guidance to put it on the carbon fiber structure of a ladder. A special screwdriver which holds the miniature screws can be inserted through a bore in the clamp and thus the module is screwed onto the structure in a fully mechanically protected way.

Although many manual steps are needed to mount the 768 modules it turned out to be very efficient and safe. Up to 40 modules could be prepared and mounted per day. Only three $(0.4 \%)$ modules have been damaged during the mounting process.

All modules are then electrically tested. This involves only short functional tests since the most likely error mode is broken wire bonds due to manual manipulations. The test steps were:

- current measurements for both low voltage and sensor bias

- scanning of the module address

- testing the module readout and measuring of the signal levels 
For layer 3 these tests can be made sector wise, i.e. 8 or 12 modules at a time. For layers 1 and 2 the tests have been made first module wise and once more sector wise after mounting the layers onto layer 3 and connecting the modules at the end flange prints. Sector wise testing of a half barrel can be done in less than an hour. At this stage exchanging or repairing of individual modules is still possible and had to be done several times. Only the completely assembled and tested half barrel is then connected to the supply tubes.

Before transporting to CERN, the system of half barrel detector and supply tube up to and including the fibre bundle pig tails have been tested extensively. Repairing of modules is now only possible with much effort and risk of further damage due to manipulation. No effort has been made to recover the $0.35 \%$ of bad channels identified in the final tests. The system has been transported to CERN in a fully functional state, without disconnecting any components.

\section{Survey}

In order to make use of the excellent spatial resolution of the detector, the absolute 3D position of each of the 768 individual modules has to be known with a precision comparable to or better than the resolution, i.e. $\lesssim 10 \mu \mathrm{m}$. This can only be achieved with track based alignment methods involving the whole tracker [4]. Here, survey information is needed for two reasons:

1. Alignment of the tracker is a highly complex minimization problem with tens of thousands of degrees of freedom. It only converges to the true minimum if the starting point is located reasonably close to it. A survey has to provide this information.

2. Any survey information, even if not absolute in space, can be used as constraints in the alignment procedure. This narrows down the phase space and improves convergence behavior.

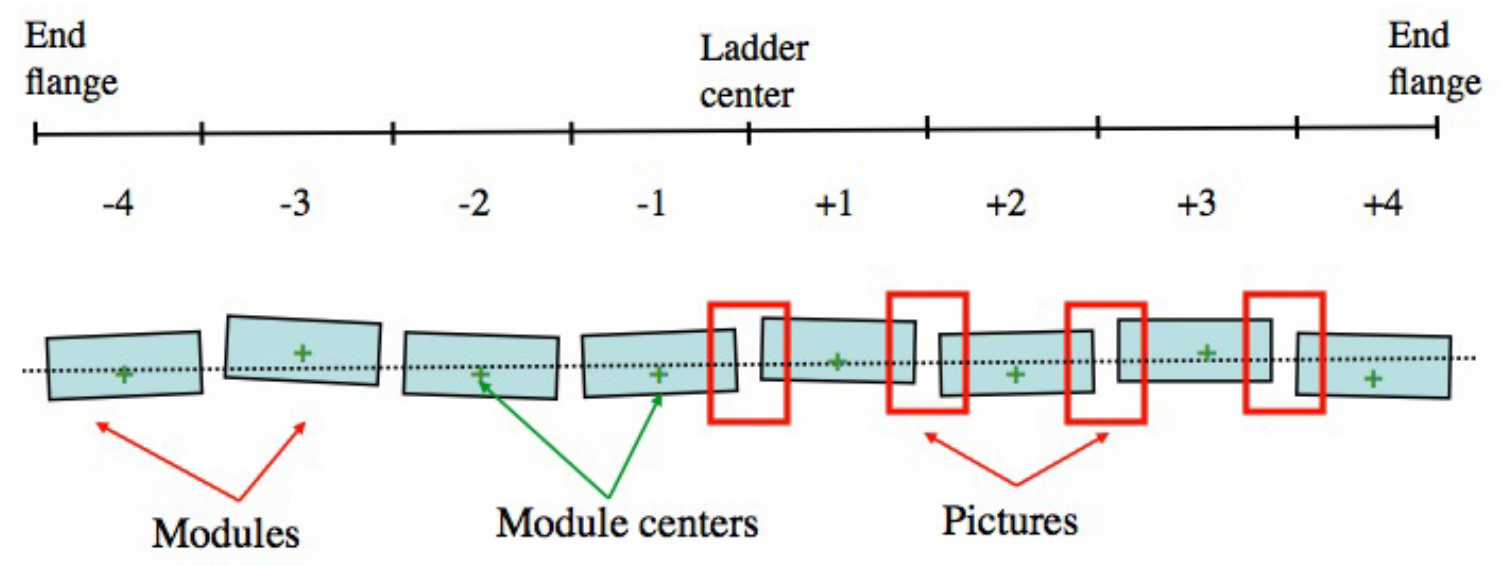

Figure 6: Principle of the survey measurement. Shown is a ladder with 8 modules (blue). Pictures (red) of the regions between modules are taken to measure module to module misalignments.

For the barrel pixel a partial survey has been done, i.e. only the position of modules with respect to ladders. The method chosen is simple, safe and gives high precision measurements in 2 
dimensions. Pictures of the region where two adjacent modules abut have been taken with a digital camera and a macro lens. This could be done on the same mounting device where the mechanical structure was fixed during module mounting. Since there are fiducial marks in each corner of the sensor this gives precise module to module measurements. This is illustrated in figure 6 . The resolution of a single alignment mark is $1.2 \mu \mathrm{m}$. However, it is only relative measurements. It connects one module to its neighbours. Starting at the ladder center this means that the errors grow toward the end of the ladder for two reasons: absolute errors add up along the axis and the errors of the inner modules lead to larger correlated errors due to a large lever arm. That way, the errors of the outermost modules become an order of magnitude larger than in the middle (see below).
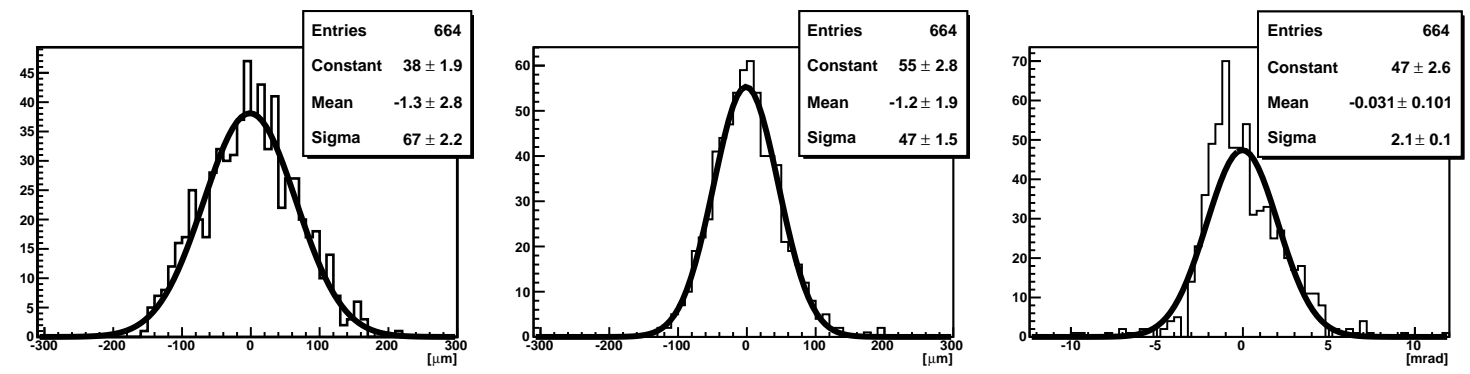

Figure 7: Measured misalignment of modules with respect to ladders. Shown are the position errors along the beam axis (left) and in $r-\phi$ (middle) and rotations around the axis perpendicular to the module plane (right).

From figure 7 we see, that the measured misalignments are quite small. It is a gaussian distribution with $\sigma=70 \mu \mathrm{m}$ along the barrel axis and $\sigma=50 \mu \mathrm{m}$ in $\mathrm{r}-\phi$. The rotations in the module planes are slightly asymmetric, with a RMS of $2.1 \mathrm{mrad}$. The error on the misalignment has to be calculated from the single point resolution taking into account all correlations. The errors in $\mathrm{r}-\phi$ are 2, 5, 11 and $20 \mu \mathrm{m}$ for the z-positions $\pm 1, \pm 2, \pm 3$ and \pm 4 respectively. The corresponding errors along the beam axis are $2,4,5.5$ and $7 \mu \mathrm{m}$.

All other movements are not measured. They are assumed as 0 with an error estimated from the mechanical assembly precision. The errors are summarized in table 1 where only the largest error along $\mathrm{z}$ has been used.

Table 1: Distributions of the remaining misalignments for all hierarchical structures. Boldface numbers have been measured in the survey. Uxxx (Gxxx) means uniform (gaussian) distribution with RMS $x x x$. The survey data for modules are 0 because the sensors are measured directly with respect to ladders, thus skipping one level of the hierarchy.

\begin{tabular}{|l|ll|ll|}
\hline Hierarchy & Without survey & With survey & \\
\hline & Translation $[\mu \mathrm{m}]$ & Rotation $[\mu \mathrm{rad}]$ & Translation $[\mu \mathrm{m}]$ & Rotation $[\mu \mathrm{rad}]$ \\
Sensor & U30 $(2 \mathrm{D})$ & - & G20 $(\mathbf{G 1 0})$ in $\mathbf{r}-\phi(\mathbf{z})$ & U110 $(2 D)$ \\
Module & U100 & U2500 & $\mathbf{0}$ & $\mathbf{0}$ \\
Ladder & U50 & - & U50 & - \\
Half-layer & U100 & - & U100 & - \\
Half-barrel & U300 & - & U300 & - \\
\hline
\end{tabular}




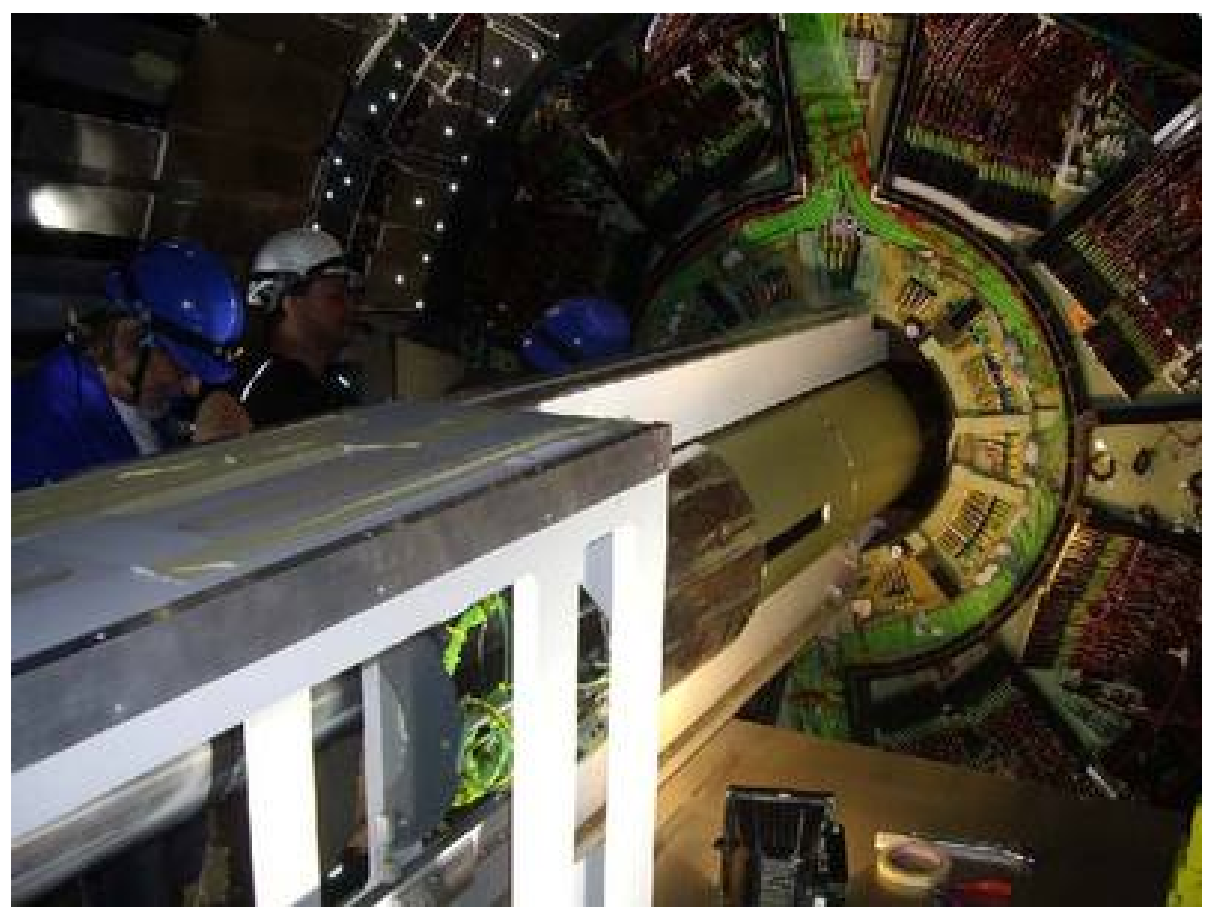

Figure 8: Insertion of the barrel pixel. Foreground: installation cassette with temporary rails. Background: patch pannel PP0 with the insertion hole. The detector is just sliding in. The green part with the slit is the supply tube with the hole for the beam pipe suspension.

\section{Insertion and commissioning}

The barrel pixel detector has been brought from PSI where it has been assembled to P5 near Cessy (F) in two parts. Each part consists of half of the barrel detector with a supply tube on each side as shown in figure 1. It has been lowered to the CMS experimental cavern July 23. Figure 8 shows the installation of the first half detector. In the foreground one can recognise the installation cassette. Temporary rails bridge the gap to the rails laid in in the pixel tracker volume. The complete system slides in with all connections in place. Connecting the power cables and the fibres at the patch panel PP0 is done in a few hours. Cleaning of the optical connectors has turned out to be the most critical and time consuming part. It followed a quick check-out where connectivity (HV and LV), clock distribution, programmability and readout signal quality has been checked. Installation and check-out took about 2 weeks.

After final testing at PSI $0.35 \%$ of the channels were broken. During transport, insertion and first check-out another $0.52 \%$ died. Since then the number of bad channels has been stable until end of the year 2008. The failure modes are:

- Modules without bias voltage (3 half and 1 full module)

- No token passage, meaning that the daisy chained module readout gets stuck (3 full modules, only $50 \%$ of each module is affected)

- Bad header in the readout data stream (2 full modules, one of them is only $50 \%$ affected) 
- No response from one module

- Individual dead readout chips (5 chips, rest of the module can still be used)

- Noisy pixels $(0.04 \%)$

The detector has then been calibrated and operated together with all CMS in cosmic data taking without and later with a magnetic field of $3.8 \mathrm{~T}$. The calibration procedure and the detector performance is described in [5].

\section{Conclusion}

After more than 10 years of development and construction, the CMS barrel pixel detector has been assembled and fully tested at PSI before transporting to CERN in July 2008. The installation and check-out has been done in two weeks. The number of bad channels is stable and less than $1 \%$. First data has been taken with cosmic muons. These tracks can already be used to align the pixel detector. Survey information taken at PSI gives important complementary information in the beginning, when statistics is too low to fully align the detector.

\section{References}

[1] CMS collaboration, 2008 JINST 3 S08004, section 3.2

[2] H.C. Kästli, et al., Nucl. Instr. and Meth. A 582 (2007) 724

[3] S. König, et al., Nucl. Instr. and Meth. A 582 (2007) 776

[4] M. Weber, et al., Nucl. Instr. and Meth. A 582 (2007) 795

[5] D. Kotlinski, Status of the CMS pixel detector, to be published 2009 in JINST, proceedings of the 2008 pixel workshop 